\title{
Thick subcategories of the stable module category
}

\author{
by
}

\author{
D. J. B en s on (Athens, Ga.), Jon F. Car ls on (Athens, Ga.) \\ and Jeremy Rickard (Bristol)
}

\begin{abstract}
We study the thick subcategories of the stable category of finitely generated modules for the principal block of the group algebra of a finite group $G$ over a field of characteristic $p$. In case $G$ is a $p$-group we obtain a complete classification of the thick subcategories. The same classification works whenever the nucleus of the cohomology variety is zero. In case the nucleus is nonzero, we describe some examples which lead us to believe that there are always infinitely many thick subcategories concentrated on each nonzero closed homogeneous subvariety of the nucleus.
\end{abstract}

1. Introduction. A subcategory of a triangulated category is said to be thick, or épaisse, if it is a triangulated subcategory and it is closed under taking direct summands. One product of the deep work of Devinatz, Hopkins and Smith [8] on stable homotopy theory was a classification of the thick subcategories of the stable homotopy category. This is described in Hopkins' survey [11], where he also states a corresponding classification of thick subcategories of the homotopy category of bounded chain complexes of finitely generated projective $R$-modules for a commutative ring $R$. In fact, this classification requires $R$ to be Noetherian, as Neeman pointed out in [13], where he also gave a complete proof.

Recent work in the modular representation theory of finite groups [3, 4, $5,18]$ has exploited the analogy between the stable homotopy category of algebraic topology and the stable module category of a finite group, which is also a triangulated category. Here we take this analogy further, and we attempt to find a classification of thick subcategories of the stable module category $\operatorname{stmod}(k G)$ of finitely generated representations of a finite group $G$ over a field $k$. Our attempt is successful when $G$ is a $p$-group, and in The-

1991 Mathematics Subject Classification: 20C20, 20J06.

The first two authors are partly supported by grants from the NSF. 
orem 3.5 we give a complete classification in terms of varieties of modules. For general groups we are not so successful, but we do get partial results in Section 5. In Section 6 we present some phenomenology in situations where our partial results do not apply, which seems to indicate that it will be difficult even to conjecture what a complete classification might be like.

Throughout this paper $k$ will be a field of characteristic $p>0$. For convenience, we shall assume that $k$ is algebraically closed.

Recall that the stable module category $\operatorname{stmod}(k G)$ has the finitely generated $k G$-modules as its objects, and a map in this category between modules $M$ and $N$ is an equivalence class of module homomorphisms, where $\alpha, \beta: M \rightarrow N$ are equivalent if the difference $\alpha-\beta$ factors through a projective module. The category $\operatorname{StMod}(k G)$ is defined similarly, except that all modules, not just finitely generated ones, are allowed. The space of maps between modules $M$ and $N$ in either of these categories is denoted by $\underline{\operatorname{Hom}}_{k G}(M, N)$. Readers not familiar with these categories may wish to consult $[4$, Section 2] and [18, Section 3].

2. Idempotent functors and varieties for infinite-dimensional modules. We shall be using recent work on "idempotent functors" and on varieties for infinite-dimensional modules, so in this section we shall summarize the facts (from [18], [4] and [5]) that will be needed.

Let $\mathcal{C}$ be a thick subcategory of $\operatorname{stmod}(k G)$ for some finite group $G$, and let $\mathcal{C}^{\oplus}$ be the smallest thick subcategory of $\operatorname{StMod}(k G)$ that contains $\mathcal{C}$ and is closed under arbitrary direct sums. As noted in the preamble to [18, Section 5], the finitely generated modules in $\mathcal{C}^{\oplus}$ are precisely those in $\mathcal{C}$.

Given a $k G$-module $X$, Section 5 of [18] describes how the techniques involved in the Brown Representability Theorem of algebraic topology can be used to construct a distinguished triangle

$$
\mathcal{T}_{\mathcal{C}}(X): \mathcal{E}_{\mathcal{C}}(X) \rightarrow X \rightarrow \mathcal{F}_{\mathcal{C}}(X) \rightarrow \Omega^{-1}\left(\mathcal{E}_{\mathcal{C}}(\mathcal{X})\right)
$$

in $\operatorname{StMod}(k G)$. Let us collect together the properties of this triangle that will be important to us.

Proposition 2.1. (a) The map $\mathcal{E}_{\mathcal{C}}(X) \rightarrow X$ is the universal map in $\operatorname{StMod}(k G)$ from an object of $\mathcal{C}^{\oplus}$ to $X$.

(b) The map $X \rightarrow \mathcal{F}_{\mathcal{C}}(X)$ is the universal map in $\operatorname{StMod}(k G)$ from $X$ to a $\mathcal{C}$-local object of $\operatorname{StMod}(k G)$ (i.e., an object $L$ for which $\underline{\operatorname{Hom}}_{k G}(C, L)=0$ for every object $C$ of $\mathcal{C}$ ).

(c) The triangle $\mathcal{T}_{\mathcal{C}}(X)$ is natural in $X$. In particular, $\mathcal{E}_{\mathcal{C}}$ and $\mathcal{F}_{\mathcal{C}}$ are functors from $\operatorname{StMod}(k G)$ to itself.

(d) The functors $\mathcal{E}_{\mathcal{C}}, \mathcal{F}_{\mathcal{C}}: \operatorname{StMod}(k G) \rightarrow \operatorname{StMod}(k G)$ are exact (i.e., they commute with $\Omega$ up to natural isomorphism and send distinguished triangles to distinguished triangles). 
(e) If $\mathcal{C}$ and $\mathcal{D}$ are two thick subcategories of $\operatorname{stmod}(k G)$ with $\mathcal{C} \subseteq \mathcal{D}$, then the map $\mathcal{E}_{\mathcal{C}} \mathcal{E}_{\mathcal{D}}(X) \rightarrow \mathcal{E}_{\mathcal{C}}(X)$, obtained by applying the functor $\mathcal{E}_{\mathcal{C}}$ to the first map in the triangle $\mathcal{T}_{\mathcal{D}}(X)$, is an isomorphism in $\operatorname{StMod}(k G)$.

(f) If $\mathcal{C}$ and $\mathcal{D}$ are two thick subcategories of $\operatorname{stmod}(k G)$, then $\mathcal{C}=\mathcal{D}$ if and only if $\mathcal{E}_{\mathcal{C}}(X) \cong \mathcal{E}_{\mathcal{D}}(X)$ for every $k G$-module $X$.

(g) If $\mathcal{C}$ has the property that $C \otimes X$ is in $\mathcal{C}$ for all objects $C$ in $\mathcal{C}$ and $X$ in $\operatorname{stmod}(k G)$, then for any object $X$ in $\operatorname{stmod}(k G), \mathcal{T}_{\mathcal{C}}(X)$ is isomorphic to the triangle obtained by tensoring $\mathcal{T}_{\mathcal{C}}(k)$ with $X$.

Proof. Parts (a) and (b) are Propositions 5.6 and 5.7 of [18], and (c) follows immediately.

Left and right adjoints to exact functors between triangulated categories are also exact (see, for example, [12, Lemma 3.9]), and so (d) follows, since $\mathcal{E}_{\mathcal{C}}$ is right adjoint to the inclusion of $\mathcal{C}^{\oplus}$ into $\operatorname{StMod}(k G)$ and $\mathcal{F}_{\mathcal{C}}$ is left adjoint to the inclusion of the full subcategory of $\mathcal{C}$-local objects into $\operatorname{StMod}(k G)$.

If $\mathcal{C} \subseteq \mathcal{D}$, then every $\mathcal{D}$-local object is also $\mathcal{C}$-local. In particular, this is true of $\mathcal{F}_{\mathcal{D}}(X)$, and so $\mathcal{E}_{\mathcal{C}} \mathcal{F}_{\mathcal{D}}(X) \cong 0$. The third object in the triangle $\mathcal{E}_{\mathcal{C}}\left(\mathcal{T}_{\mathcal{D}}(X)\right)$ is therefore zero, and so the first map, $\mathcal{E}_{\mathcal{C}} \mathcal{E}_{\mathcal{D}}(X) \rightarrow \mathcal{E}_{\mathcal{C}}(X)$, is an isomorphism. Therefore (e) is true.

If $\mathcal{C}=\mathcal{D}$, then certainly $\mathcal{E}_{\mathcal{C}}(X) \cong \mathcal{E}_{\mathcal{D}}(X)$ for any $X$. Conversely, if $\mathcal{E}_{\mathcal{C}}(X) \cong \mathcal{E}_{\mathcal{D}}(X)$ for every $X$, then

$$
X \text { is in } \mathcal{C}^{\oplus} \Leftrightarrow X \cong \mathcal{E}_{\mathcal{C}}(X) \Leftrightarrow X \cong \mathcal{E}_{\mathcal{D}}(X) \Leftrightarrow X \text { is in } \mathcal{D}^{\oplus} \text {. }
$$

Thus $\mathcal{C}^{\oplus}=\mathcal{D}^{\oplus}$, and so, considering the finitely generated modules in these categories, $\mathcal{C}=\mathcal{D}$. So (f) is true.

Part $(\mathrm{g})$ is Proposition 5.13 of [18].

The modules $\mathcal{E}_{\mathcal{C}}(X)$ and $\mathcal{F}_{\mathcal{C}}(X)$ will usually not be finitely generated, even if $X$ is, and so the use of idempotent functors forces us to consider infinitely generated modules. For this reason, we shall need to use the theory of "varieties" for infinitely generated modules, which was recently developed in $[4,5]$. So let us now collect together the facts that we need from this theory.

We shall assume the reader has some familiarity with the theory of varieties for finitely generated modules, where a closed homogeneous subvariety $V_{G}(M)$ of the maximal ideal spectrum $V_{G}(k)$ of the cohomology ring $H^{*}(G, k)$ is associated to each finitely generated $k G$-module $M$. A fairly comprehensive exposition can be found in Chapter 5 of [2].

In what seems to be the most useful generalization of this theory to modules that are not necessarily finitely generated, the "variety" of a module $M$ is not really a variety, but a set $\mathcal{V}_{G}(M)$ of closed homogeneous irreducible nonzero subvarieties of $V_{G}(k)$ (or, equivalently, a subset of the scheme Proj $\left.H^{*}(G, k)\right)$. We refer to [5, Definition 10.2] for the precise definition of $\mathcal{V}_{G}(M)$; here we just recall some of the more important properties. 
Proposition 2.2. (a) The "variety" $\mathcal{V}_{G}(k)$ of the trivial $k G$-module $k$ is the set of all closed homogeneous irreducible nonzero subvarieties of $V_{G}(k)$.

(b) $A k G$-module $M$ is projective if and only if $\mathcal{V}_{G}(M)=\emptyset$.

(c) If $M$ is a finitely generated $k G$-module, then

$$
\mathcal{V}_{G}(M)=\left\{V \in \mathcal{V}_{G}(k): V \subseteq V_{G}(M)\right\} .
$$

(d) If

$$
M_{1} \rightarrow M_{2} \rightarrow M_{3} \rightarrow \Omega^{-1}\left(M_{1}\right)
$$

is a distinguished triangle in $\operatorname{StMod}(k G)$, then

$$
\mathcal{V}_{G}\left(M_{i}\right) \subseteq \mathcal{V}_{G}\left(M_{j}\right) \cup \mathcal{V}_{G}\left(M_{k}\right)
$$

for $\{i, j, k\}=\{1,2,3\}$.

(e) For any family $\left\{M_{i}: i \in I\right\}$ of $k G$-modules,

$$
\mathcal{V}_{G}\left(\bigoplus_{i \in I} M_{i}\right)=\bigcup_{i \in I}\left(\mathcal{V}_{G}\left(M_{i}\right)\right)
$$

(f) (Tensor Product Theorem) For all $k G$-modules $M$ and $N$,

$$
\mathcal{V}_{G}\left(M \otimes_{k} N\right)=\mathcal{V}_{G}(M) \cap \mathcal{V}_{G}(N) \text {. }
$$

(g) Let $W$ be a closed homogeneous subvariety of $V_{G}(k)$, and let $\mathcal{C}(W)$ be the thick subcategory of $\operatorname{stmod}(k G)$ consisting of the finitely generated modules $M$ with $V_{G}(M) \subseteq W$. If a $k G$-module $N$ is in $\mathcal{C}(W)^{\oplus}$, then

$$
\mathcal{V}_{G}(N) \subseteq\left\{V \in \mathcal{V}_{G}(k): V \subseteq W\right\} .
$$

Proof. All of these facts follow easily from what is contained in [5].

As noted after Definition 10.2 of [5], (a) follows immediately from the definition and [5, Lemma 10.2(ii)].

Also implicit in [5] is (b). For an elementary abelian $p$-group, it follows from [5, Corollary 5.6 and Theorem 10.5]. For a general group $G$, it then follows by Chouinard's Theorem [7] and [5, Theorem 10.6].

Using [5, Theorem 10.6] again, the proof of (c) can be reduced to the case of an elementary abelian $p$-group, when it follows from [5, Remark 5.5(ii) and Theorem 10.5].

Both (d) and (e) can be proved using [5, Lemma 10.3], which says that for any $V \in \mathcal{V}_{G}(k)$ there is a certain module $\kappa(V)$ such that, for any $k G$-module $M, V \in \mathcal{V}_{G}(M)$ if and only if $\kappa(V) \otimes_{k} M$ is not projective.

The Tensor Product Theorem (f) is Theorem 10.8 of [5].

Finally, (g) is an immediate consequence of (d) and (e).

3. Thick subcategories with ideal closure. If $G$ is a finite group and $W$ is a closed homogeneous subvariety of the maximal ideal spectrum $V_{G}(k)$ of $H^{*}(G, k)$, then one of the more interesting thick subcategories of $\operatorname{stmod}(k G)$ is $\mathcal{C}(W)$, the full subcategory consisting of modules whose 
varieties are contained in $W$. More general examples of thick subcategories can be defined in terms of varieties as follows.

Definition 3.1. Let $G$ be a finite group, and let $\mathcal{X}$ be a nonempty set of closed homogeneous subvarieties of $V_{G}(k)$ that is closed under specialization (i.e., if $W \in \mathcal{X}$ and $W^{\prime} \subseteq W$, then $W^{\prime} \in \mathcal{X}$ ) and finite unions. Then $\mathcal{C}(\mathcal{X})$ is the thick subcategory of $\operatorname{stmod}(k G)$ consisting of modules $M$ with $V_{G}(M) \in \mathcal{X}$.

It follows easily from standard properties of varieties for modules that $\mathcal{C}(\mathcal{X})$ is indeed a thick subcategory. If $\mathcal{X}$ has a maximal element $W$, then $\mathcal{C}(\mathcal{X})$ coincides with the thick subcategory $\mathcal{C}(W)$ described above. Also, if $\mathcal{X}$ is the set of all closed homogeneous subvarieties of $V_{G}(k)$ of dimension less than $c$ for some positive integer $c$, then $\mathcal{C}(\mathcal{X})$ is just the thick subcategory of all finitely generated modules with complexity less than $c$. Many familiar examples of thick subcategories are therefore subsumed by this definition.

We now consider thick subcategories of $\operatorname{stmod}(k G)$ which have ideal closure as defined below. It will be shown that every such thick subcategory has the form $\mathcal{C}(\mathcal{X})$ for some $\mathcal{X}$. Most importantly, all thick subcategories of $\operatorname{stmod}(k G)$ for $G$ a $p$-group have ideal closure, and hence we have a complete classification of the thick subcategories in this case.

Definition 3.2. A thick subcategory $\mathcal{C}$ of $\operatorname{stmod}(k G)$ is said to have ideal closure if $C \otimes X$ is in $\mathcal{C}$ for any $C$ in $\mathcal{C}$ and $X$ in $\operatorname{stmod}(k G)$.

The following proposition is the key step in the proof of the main results of the section.

Proposition 3.3. Let $M$ be a finitely generated $k G$-module, and let $W=V_{G}(M)$. Let $\langle\langle M\rangle\rangle$ be the thick subcategory of $\operatorname{stmod}(k P)$ generated by $M \otimes X$ for all $X$ in $\operatorname{stmod}(k G)$. Then $\langle\langle M\rangle\rangle$ is equal to $\mathcal{C}(W)$.

Proof. Since $M$ is in $\mathcal{C}(W)$, it is clear that $\langle\langle M\rangle\rangle$ is contained in $\mathcal{C}(W)$. Therefore by Proposition 2.1 the functors $\mathcal{E}_{\langle\langle M\rangle\rangle} \mathcal{E}_{W}$ and $\mathcal{E}_{\langle\langle M\rangle\rangle}$ are isomorphic. Hence, for any $k G$-module $N$ the distinguished triangle $\mathcal{T}_{\langle\langle M\rangle\rangle}\left(\mathcal{E}_{W}(N)\right)$ is of the form

$$
\mathcal{E}_{\langle\langle M\rangle\rangle}(N) \rightarrow \mathcal{E}_{W}(N) \rightarrow \mathcal{F}_{\langle\langle M\rangle\rangle} \mathcal{E}_{W}(N) \rightarrow \Omega^{-1}\left(\mathcal{E}_{\langle\langle M\rangle\rangle}(N)\right) .
$$

We shall prove that $\mathcal{F}_{\langle\langle M\rangle\rangle} \mathcal{E}_{W}(N)$ is projective, and so the first map in this triangle is a stable isomorphism between $\mathcal{E}_{\langle\langle M\rangle\rangle}(N)$ and $\mathcal{E}_{W}(N)$.

The first two terms of the triangle $\mathcal{T}_{\langle\langle M\rangle\rangle}\left(\mathcal{E}_{W}(N)\right)$ are in $\mathcal{C}(W)^{\oplus}$, and therefore so is $\mathcal{F}_{\langle\langle M\rangle\rangle} \mathcal{E}_{W}(N)$. Hence,

$$
\mathcal{V}_{G}\left(\mathcal{F}_{\langle\langle M\rangle\rangle} \mathcal{E}_{W}(N)\right) \subseteq\left\{V \in \mathcal{V}_{G}(k): V \subseteq W\right\}=\mathcal{V}_{G}(M),
$$

by Proposition 2.2 . 
Since $\mathcal{F}_{\langle\langle M\rangle\rangle} \mathcal{E}_{W}(N)$ is $\langle\langle M\rangle\rangle$-local and $M$ is finitely generated,

$$
0=\underline{\operatorname{Hom}}_{k G}\left(S \otimes M, \mathcal{F}_{\langle\langle M\rangle\rangle} \mathcal{E}_{W}(N)\right) \cong \underline{\operatorname{Hom}}_{k G}\left(S, M^{*} \otimes_{k} \mathcal{F}_{\langle\langle M\rangle\rangle} \mathcal{E}_{W}(N)\right)
$$

for all simple $k G$-modules $S$ (and where $M^{*}$ denotes the $k$-linear dual of $M)$. This implies that $M^{*} \otimes_{k} \mathcal{F}_{\langle\langle M\rangle\rangle} \mathcal{E}_{W}(N)$ is projective, and so, by the tensor product theorem for varieties (see Proposition 2.2),

$$
\begin{aligned}
\emptyset & =\mathcal{V}_{G}\left(M^{*} \otimes_{k} \mathcal{F}_{\langle\langle M\rangle\rangle} \mathcal{E}_{W}(N)\right) \\
& =\mathcal{V}_{G}(M) \cap \mathcal{V}_{G}\left(\mathcal{F}_{\langle\langle M\rangle\rangle} \mathcal{E}_{W}(N)\right)=\mathcal{V}_{G}\left(\mathcal{F}_{\langle\langle M\rangle\rangle} \mathcal{E}_{W}(N)\right) .
\end{aligned}
$$

Thus $\mathcal{F}_{\langle\langle M\rangle\rangle} \mathcal{E}_{W}(N)$ is projective, and so $\mathcal{E}_{\langle\langle M\rangle\rangle}(N)$ and $\mathcal{E}_{W}(N)$ are stably isomorphic. Hence $\mathcal{C}(W)$ and $\langle\langle M\rangle\rangle$ are equal, by Proposition 2.1.

The classification of thick subcategories with ideal closure in $\operatorname{stmod}(k G)$ now follows easily.

TheOREM 3.4. Suppose that $\mathcal{C}$ is a thick subcategory with ideal closure in $\operatorname{stmod}(k G)$. Then $\mathcal{C}=\mathcal{C}(\mathcal{X})$ for some nonempty set $\mathcal{X}$ of closed homogeneous subvarieties of $V_{G}(k)$ closed under specialization and finite unions.

Pr o of. Suppose that $\mathcal{X}$ is the set of subvarieties of $V_{G}(k)$ that occur as $V_{G}(M)$ for some object $M$ of $\mathcal{C}$. Then $\mathcal{X}$ is nonempty, since the zero module is in $\mathcal{C}$, and is closed under finite unions because $\mathcal{C}$ is closed under finite direct sums. It is well known that every closed homogeneous subvariety $W^{\prime}$ of $V_{G}(k)$ occurs as the variety of some finitely generated $k G$-module $L$ (see, for example, [2, Corollary 5.9.2]). Therefore, if $W^{\prime}$ is a subvariety of $V_{G}(M)$ for some $M$ in $\mathcal{C}$, then $M \otimes_{k} L$ has variety $W^{\prime}$ and is in the thick subcategory $\langle\langle M\rangle\rangle$. In particular, it is in $\mathcal{C}$. Hence $\mathcal{X}$ is closed under specialization.

Clearly, $\mathcal{C}$ is contained in $\mathcal{C}(\mathcal{X})$. But also, for each object $N$ of $\mathcal{C}(\mathcal{X})$, there is an object $N^{\prime}$ of $\mathcal{C}$ with $V_{G}\left(N^{\prime}\right)=V_{G}(N)$. By Proposition 3.3, $N$ and $N^{\prime}$ generate the same thick subcategory, and so $N$ must be in $\mathcal{C}$. Hence $\mathcal{C}=\mathcal{C}(\mathcal{X})$

Corollary 3.5. Let $P$ be a p-group, and let $\mathcal{C}$ be a thick subcategory of $\operatorname{stmod}(k P)$. Then $\mathcal{C}=\mathcal{C}(\mathcal{X})$ for some nonempty set $\mathcal{X}$ of closed homogeneous subvarieties of $V_{P}(k)$ closed under specialization and finite unions.

Proof. The important thing to note here is that $\operatorname{stmod}(k P)=\langle k\rangle$, the thick subcategory generated by the trivial module $k$. Also, if $C$ is an object in $\mathcal{C}$ and $X$ is in $\langle k\rangle$ then $C \otimes X$ is in $\mathcal{C}$. So $\mathcal{C}$ has ideal closure.

4. The nucleus and modules with no cohomology. For a general finite group $G$, the modules in the principal block form a proper thick subcategory of $\operatorname{stmod}(k G)$. More interestingly, perhaps, the modules $M$ in the 
principal block for which the Tate cohomology $\widehat{H}^{*}(G, M)$ vanishes in all degrees form a thick subcategory that is not usually trivial. Such modules were studied in [6], where several conjectures were proposed that were settled recently in [3] using some of the theory of idempotent functors described in Section 2. In the next section we shall need to use this work, so let us give a brief summary of some of the important points.

One of the important theorems is a classification of the finite groups $G$ for which $\widehat{H}^{*}(G, M) \neq 0$ for all nonprojective modules $M$ in the principal block. In [6, Section 2], the term trivial homology module is introduced. It follows immediately from what is proved in [6, Sections 2 and 3] about these modules that they are precisely the modules that are in the triangulated subcategory of $\operatorname{stmod}(k G)$ generated by the trivial module. In [3, Theorem 1.4] the following result was proved, which had previously been established in odd characteristic in $[6]$.

Proposition 4.1. Let $G$ be a finite group. Then the following are equivalent:

(a) The full subcategory of $\operatorname{stmod}(k G)$ consisting of modules in the principal block is generated, as a triangulated category, by the trivial module $k$.

(b) If $M$ is a finitely generated $k G$-module for which $\widehat{H}^{*}(G, M)=0$, then no nonprojective direct summand of $M$ belongs to the principal block.

(c) Every element of order $p$ in $G$ has a p-nilpotent centralizer.

In fact, [3, Theorem 1.4] gives twelve equivalent conditions.

To say something about groups that do not satisfy the conditions of Proposition 4.1, Benson, Carlson and Robinson introduced a closed homogeneous subvariety $Y_{G}$ of $V_{G}(k)$ called the nucleus. Let us recall the definition.

Definition $4.2([6])$. Let $G$ be a finite group. The nucleus $Y_{G}$ is the union of the images of the restriction maps

$$
\operatorname{res}_{G, H}^{*}: V_{H}(k) \rightarrow V_{G}(k),
$$

as $H$ runs over the set of subgroups of $G$ for which the centralizer $C_{G}(H)$ is not $p$-nilpotent. (Except that if $G$ is $p$-nilpotent, then $Y_{G}$ should be taken to be $\{0\}$ rather than $\emptyset$.)

Note that $Y_{G}=\{0\}$ if and only if the equivalent conditions of Proposition 4.1 hold.

The nucleus was introduced in order to state the following result, conjectured in [6] and proved in [3].

Proposition 4.3. Let $G$ be a finite group. Every finitely generated module in the principal block $B_{0}(k G)$ is contained in $\left\langle k, \mathcal{C}\left(Y_{G}\right)\right\rangle$, the thick subcategory of $\operatorname{stmod}(k G)$ generated by the trivial module $k$ together with all the modules whose variety is contained in the nucleus. 
The thick subcategory $\left\langle k, \mathcal{C}\left(Y_{G}\right)\right\rangle$ consists of the direct summands of what are called nuclear homology modules in $[6,3]$, so this is just a restatement of [3, Theorem 1.2].

Proposition 4.4. Let $G$ be a finite group. If $M$ is a module in the principal block such that $\widehat{H}^{*}(G, M)=0$, then $M$ is in $\mathcal{C}\left(Y_{G}\right)^{\oplus}$.

If $M$ is finitely generated, then the conclusion is just that $V_{G}(M) \subseteq Y_{G}$, and this was shown in [6, Corollary 10.12] to be a consequence of Proposition 4.3. We shall give a proof using idempotent functors that does not require $M$ to be finitely generated.

Pr o of. Consider the distinguished triangle $\mathcal{T}_{\mathcal{C}\left(Y_{G}\right)}(M)$. By [18, Proposition 5.13], this is isomorphic to the triangle obtained by taking the tensor product of $M$ with $\mathcal{T}_{\mathcal{C}\left(Y_{G}\right)}(k)$. Also, the construction of $\mathcal{T}_{\mathcal{C}\left(Y_{G}\right)}(k)$ in [18, Section 6] makes it clear that $\mathcal{E}_{\mathcal{C}\left(Y_{G}\right)}(k)$ and $\mathcal{F}_{\mathcal{C}\left(Y_{G}\right)}(k)$ are in $\langle k\rangle^{\oplus}$. It follows that $\mathcal{E}_{\mathcal{C}\left(Y_{G}\right)}(M)$ and $\mathcal{F}_{\mathcal{C}\left(Y_{G}\right)}(M)$ are in $\langle M\rangle^{\oplus}$, and, in particular, are in the category of $\langle k\rangle$-local modules of the principal block.

Since $\mathcal{F}_{\mathcal{C}\left(Y_{G}\right)}(M)$ is also $\mathcal{C}\left(Y_{G}\right)$-local, it follows from Proposition 4.3 that it must be projective, and so

$$
M \cong \mathcal{E}_{\mathcal{C}\left(Y_{G}\right)}(M),
$$

which is in $\mathcal{C}\left(Y_{G}\right)^{\oplus}$.

We shall also need the following consequence of Proposition 4.3.

Proposition 4.5. Let $G$ be a finite group, and let $M$ be a finitely generated $k G$-module in the principal block for which

$$
V_{G}(M) \cap Y_{G}=\{0\} .
$$

Then $M$ is in the thick subcategory $\langle k\rangle$ of $\operatorname{stmod}(k G)$ generated by the trivial module $k$.

P r o of. Consider the distinguished triangle

$$
\mathcal{T}_{\langle k\rangle}(M): \mathcal{E}_{\langle k\rangle}(M) \rightarrow M \rightarrow \mathcal{F}_{\langle k\rangle}(M) \rightarrow \Omega^{-1}\left(\mathcal{E}_{\langle k\rangle}(M)\right) .
$$

The third term, $\mathcal{F}_{\langle k\rangle}(M)$, is $\langle k\rangle$-local, and so, by Propositions 4.4 and 2.2,

$$
\mathcal{V}_{G}\left(\mathcal{F}_{\langle k\rangle}(M)\right) \subseteq\left\{W \in \mathcal{V}_{G}(k): W \subseteq Y_{G}\right\} .
$$

Since $M$ is finitely generated, it follows from the Tensor Product Theorem of Proposition 2.2 that

$$
\mathcal{V}_{G}\left(\operatorname{Hom}_{k}\left(M, \mathcal{F}_{\langle k\rangle}(M)\right)\right)=\emptyset,
$$

and so

$$
\underline{\operatorname{Hom}}_{k G}\left(M, \mathcal{F}_{\langle k\rangle}(M)\right)=0
$$


By the universal property of the second map in the triangle $\mathcal{T}_{\langle k\rangle}(M)$, it follows that $\mathcal{F}_{\langle k\rangle}(M)$ is projective, and so

$$
M \cong \mathcal{E}_{\langle k\rangle}(M),
$$

which is in $\langle k\rangle^{\oplus}$. Since $M$ is finitely generated, it must be in $\langle k\rangle$.

5. Thick subcategories in the principal block. In this section, we shall give some generalizations of Corollary 3.5, the classification of thick subcategories of $\operatorname{stmod}(k P)$ for a $p$-group $P$, to more general groups. If $G$ is a group for which $k G$ has more than one block, then the modules belonging to a particular block form a thick subcategory. We shall restrict our attention to modules that belong to the principal block $B_{0}(k G)$, although there are doubtless things that could be said about other blocks. Even with this restriction, the thick subcategories cannot, in general, be classified in terms of varieties in the same way as they could for $p$-groups: for example, the thick subcategory $\langle k\rangle$ generated by the trivial module contains modules with all possible varieties, but it does not coincide with the thick subcategory of all modules in the principal block unless the nucleus $Y_{G}$ is trivial. What we shall show is that, in a sense, the nucleus is the only obstruction to a full generalization: if we restrict our attention to thick subcategories containing no nonprojective modules whose variety is in the nucleus, or containing all modules with variety in the nucleus, then we do get a satisfactory classification. In particular, Corollary 3.5 generalizes to groups which have a trivial nucleus.

Since we shall be considering only modules in the principal block, let us introduce the following notation for various thick subcategories.

DeFinition 5.1. Let $G$ be a finite group.

(a) We shall denote by $\operatorname{stmod}_{0}(k G)$ the thick subcategory of $\operatorname{stmod}(k G)$ consisting of the finitely generated $k G$-modules that belong to the principal block $B_{0}(k G)$. Similarly, $\operatorname{StMod}_{0}(k G)$ is the thick subcategory of $\operatorname{StMod}(k G)$ consisting of all modules that belong to the principal block.

(b) If $W$ is a closed homogeneous subvariety of $V_{G}(k)$, then $\mathcal{C}_{0}(W)$ is the thick subcategory of $\operatorname{stmod}(k G)$ consisting of modules in $\mathcal{C}(W)$ that belong to the principal block.

(c) If $\mathcal{X}$ is a nonempty family of closed homogeneous subvarieties of $V_{G}(k)$ that is closed under specialization and finite unions, then $\mathcal{C}_{0}(\mathcal{X})$ is the thick subcategory of $\operatorname{stmod}(k G)$ consisting of modules in $\mathcal{C}(\mathcal{X})$ that belong to the principal block.

Let us start with some easy lemmas regarding idempotent functors and tensor products as applied to modules in the principal block. 
Lemma 5.2. Let $G$ be a finite group, let $\mathcal{C}$ be a thick subcategory of the category $\operatorname{stmod}_{0}(k G)$, and let $N$ be a $k G$-module. Then $\mathcal{E}_{\mathcal{C}}(N)$ is in $\operatorname{StMod}_{0}(k G)$. If $N$ is in $\operatorname{StMod}_{0}(k G)$, then so is $\mathcal{F}_{\mathcal{C}}(N)$.

Pr o o f. Since $\operatorname{StMod}_{0}(k G)$ contains $\mathcal{C}$ and is closed under arbitrary direct sums, it contains $\mathcal{C}^{\oplus}$, of which $\mathcal{E}_{\mathcal{C}}(N)$ is an object.

The first term of the distinguished triangle

$$
\mathcal{E}_{\mathcal{C}}(N) \rightarrow N \rightarrow \mathcal{F}_{\mathcal{C}}(N) \rightarrow \Omega^{-1}\left(\mathcal{E}_{\mathcal{C}}(N)\right)
$$

is in $\operatorname{StMod}_{0}(k G)$, and so if either $N$ or $\mathcal{F}_{\mathcal{C}}(N)$ is in $\operatorname{StMod}_{0}(k G)$, so is the other.

LEMMA 5.3. Let $G$ be a finite group, and let $\mathcal{C}$ be a thick subcategory of $\operatorname{StMod}_{0}(k G)$. Let $N$ be a module in $\mathcal{C}$, and let $M$ be a finitely generated $k G$-module. If $M$ is in the thick subcategory $\langle k\rangle$ generated by the trivial module, then $M \otimes_{k} N$ and $\operatorname{Hom}_{k}(M, N)$ are both in $\mathcal{C}$. In particular, if $M$ is in $\operatorname{stmod}_{0}(k G)$ and $V_{G}(M) \cap Y_{G}=\{0\}$, then $M \otimes_{k} N$ and $\operatorname{Hom}_{k}(M, N)$ are both in $\mathcal{C}$.

Pr o o f. Let $\mathcal{D}$ be the class of finitely generated $k G$-modules $X$ for which $X \otimes_{k} N$ and $\operatorname{Hom}_{k}(X, N)$ are both in $\mathcal{C}$. Then $\mathcal{D}$ forms a thick subcategory of $\operatorname{stmod}(k G)$ which contains the trivial module $k$, and so it must contain all of $\langle k\rangle$. The final statement follows because if $M$ is in $\operatorname{stmod}_{0}(k G)$ and $V_{G}(M) \cap Y_{G}=\{0\}$, then $M$ is in $\langle k\rangle$, by Proposition 4.5.

Let us start by studying thick subcategories of $\operatorname{stmod}_{0}(k G)$ consisting entirely of modules whose variety intersects the nucleus $Y_{G}$ trivially. As in the case of $p$-groups, the key step in the classification of such subcategories consists of identifying the thick subcategory generated by a single module. We have the following analogue of Proposition 3.3.

Proposition 5.4. Let $G$ be a finite group, and let $M$ be a $k G$-module in $\operatorname{stmod}_{0}(k G)$ whose variety $V_{G}(M)=W$ satisfies $W \cap Y_{G}=\{0\}$. Then the thick subcategories $\langle M\rangle$ and $\mathcal{C}_{0}(W)$ coincide.

Proof. If $N$ is any $k G$-module, then the distinguished triangle $\mathcal{T}_{\langle M\rangle}\left(\mathcal{E}_{\mathcal{C}_{0}(W)}(N)\right)$ has the form

$$
\mathcal{E}_{\langle M\rangle}(N) \rightarrow \mathcal{E}_{\mathcal{C}_{0}(W)}(N) \rightarrow \mathcal{F}_{\langle M\rangle} \mathcal{E}_{\mathcal{C}_{0}(W)}(N) \rightarrow \Omega^{-1}\left(\mathcal{E}_{\langle M\rangle}(N)\right) .
$$

As in the proof of Proposition 3.3, we shall show that $\mathcal{E}_{\langle M\rangle}(N)$ and $\mathcal{E}_{\mathcal{C}_{0}(W)}(N)$ are stably isomorphic (and so $\langle M\rangle=\mathcal{C}_{0}(W)$, by Proposition 2.1) by showing that $\mathcal{F}_{\langle M\rangle} \mathcal{E}_{\mathcal{C}_{0}(W)}(N)$ is projective.

Since the first two terms of the triangle are in $\mathcal{C}_{0}(W)^{\oplus}$, so is the third. Therefore $\mathcal{F}_{\langle M\rangle} \mathcal{E}_{\mathcal{C}_{0}(W)}(N)$ is in $\operatorname{StMod}_{0}(k G)$ and

$$
\mathcal{V}_{G}\left(\mathcal{F}_{\langle M\rangle} \mathcal{E}_{\mathcal{C}_{0}(W)}(N)\right) \subseteq\left\{V \in \mathcal{V}_{G}(k): V \subseteq W\right\}=\mathcal{V}_{G}(M)
$$


Because $\mathcal{F}_{\langle M\rangle} \mathcal{E}_{\mathcal{C}_{0}(W)}(N)$ is $\langle M\rangle$-local, we have, for every $n \in \mathbb{Z}$,

$$
0=\underline{\operatorname{Hom}}_{k G}\left(\Omega^{n} M, \mathcal{F}_{\langle M\rangle} \mathcal{E}_{\mathcal{C}_{0}(W)}(N)\right) \cong \widehat{H}^{n}\left(G, M^{*} \otimes_{k} \mathcal{F}_{\langle M\rangle} \mathcal{E}_{\mathcal{C}_{0}(W)}(N)\right) .
$$

But, by Lemma 5.3, $M^{*} \otimes_{k} \mathcal{F}_{\langle M\rangle} \mathcal{E}_{\mathcal{C}_{0}(W)}(N)$ is in $\operatorname{StMod}_{0}(k G)$, its variety is contained in $\mathcal{V}_{G}(M)$ by Proposition 2.2(f), and so, by Proposition 4.5, it must be projective. Hence,

$$
\begin{aligned}
\emptyset & =\mathcal{V}_{G}\left(M^{*} \otimes_{k} \mathcal{F}_{\langle M\rangle} \mathcal{E}_{\mathcal{C}_{0}(W)}(N)\right) \\
& =\mathcal{V}_{G}(M) \cap \mathcal{V}_{G}\left(\mathcal{F}_{\langle M\rangle} \mathcal{E}_{\mathcal{C}_{0}(W)}(N)\right)=\mathcal{V}_{G}\left(\mathcal{F}_{\langle M\rangle} \mathcal{E}_{\mathcal{C}_{0}(W)}(N)\right) .
\end{aligned}
$$

Thus $\mathcal{F}_{\langle M\rangle} \mathcal{E}_{\mathcal{C}_{0}(W)}(N)$ is projective, as required.

The following partial classification of thick subcategories for a general group $G$ follows from the previous result in the same way that Theorem 3.4 and Corollary 3.5 followed from Proposition 3.3.

THEOREM 5.5. Let $G$ be a finite group, and let $\mathcal{C}$ be a thick subcategory of $\operatorname{stmod}_{0}(k G)$ containing only modules $M$ for which $V_{G}(M) \cap Y_{G}=\{0\}$. Then $\mathcal{C}=\mathcal{C}_{0}(\mathcal{X})$ for some nonempty set $\mathcal{X}$ of closed homogeneous subvarieties of $V_{G}(k)$, closed under specialization and finite unions, such that

$$
W \in \mathcal{X} \Rightarrow W \cap Y_{G}=\{0\} .
$$

Using the classification of groups $G$ for which the nucleus $Y_{G}$ is trivial, the following corollary follows immediately.

COROLlaRY 5.6. Let $G$ be a finite group in which the centralizers of all elements of order $p$ are p-nilpotent. Then every thick subcategory of $\operatorname{stmod}_{0}(k G)$ is of the form $\mathcal{C}_{0}(\mathcal{X})$ for some nonempty set $\mathcal{X}$ of closed homogeneous subvarieties of $V_{G}(k)$, closed under specialization and finite unions.

In a slightly different direction, we can use the same methods to classify thick subcategories of $\operatorname{stmod}_{0}(k G)$ that contain $\mathcal{C}_{0}\left(Y_{G}\right)$. Of course, this is equivalent to classifying thick subcategories of the quotient category $\operatorname{stmod}_{0}(k G) / \mathcal{C}_{0}\left(Y_{G}\right)$. The analogue of Proposition 3.3 that we need is as follows.

Proposition 5.7. Let $G$ be a finite group, let $M$ be a $k G$-module in the category $\operatorname{stmod}_{0}(k G)$, and let $W=V_{G}(M)$. Then $\mathcal{Y}_{0}(M)$, the thick subcategory of $\operatorname{stmod}_{0}(k G)$ generated by $\mathcal{C}_{0}\left(Y_{G}\right)$ together with $M$, is equal to $\mathcal{C}_{0}\left(Y_{G} \cup W\right)$.

Proof. Let us start by choosing some module $Y$ in $\operatorname{stmod}_{0}(k G)$ with $V_{G}(Y)=Y_{G}$.

For any $k G$-module $N$, the distinguished triangle $\mathcal{T}_{\mathcal{Y}_{0}(M)}\left(\mathcal{E}_{\mathcal{C}_{0}\left(Y_{G} \cup W\right)}(N)\right)$ has the form

$$
\mathcal{E}_{\mathcal{Y}_{0}(M)}(N) \rightarrow \mathcal{E}_{\mathcal{C}_{0}\left(Y_{G} \cup W\right)}(N) \rightarrow \mathcal{F}_{\mathcal{Y}_{0}(M)} \mathcal{E}_{\mathcal{C}_{0}\left(Y_{G} \cup W\right)}(N) \rightarrow \Omega^{-1}\left(\mathcal{E}_{\mathcal{Y}_{0}(M)}(N)\right) .
$$


As in the proofs of Propositions 3.3 and 5.4, we just have to show that the third term of this triangle is projective.

Certainly, $\mathcal{V}_{G}\left(\mathcal{F}_{\mathcal{Y}_{0}(M)} \mathcal{E}_{\mathcal{C}_{0}\left(Y_{G} \cup W\right)}(N)\right)$ is contained in $\mathcal{V}_{G}(M \oplus Y)$, since this is true of the first two terms of the triangle.

But, since $M \oplus Y$ is in $\mathcal{Y}_{0}(M), \mathcal{F}_{\mathcal{Y}_{0}(M)} \mathcal{E}_{\mathcal{C}_{0}\left(Y_{G} \cup W\right)}(N)$ is $(M \oplus Y)$-local, and so, for any $n \in \mathbb{Z}$,

So

$$
\begin{aligned}
0 & =\operatorname{Hom}\left(\Omega^{n}(M \oplus Y), \mathcal{F}_{\mathcal{Y}_{0}(M)} \mathcal{E}_{\mathcal{C}_{0}\left(Y_{G} \cup W\right)}(N)\right) \\
& \cong \widehat{H}^{n}\left(G,(M \oplus Y)^{*} \otimes_{k} \mathcal{F}_{\mathcal{Y}_{0}(M)} \mathcal{E}_{\mathcal{C}_{0}\left(Y_{G} \cup W\right)}(N)\right) .
\end{aligned}
$$

$$
\begin{aligned}
\mathcal{V}_{G}\left(\mathcal{F}_{\mathcal{Y}_{0}(M)} \mathcal{E}_{\mathcal{C}_{0}\left(Y_{G} \cup W\right)}(N)\right) & =\mathcal{V}_{G}(M \oplus Y) \cap \mathcal{V}_{G}\left(\mathcal{F}_{\mathcal{Y}_{0}(M)} \mathcal{E}_{\mathcal{C}_{0}\left(Y_{G} \cup W\right)}(N)\right) \\
& =\mathcal{V}_{G}\left((M \oplus Y)^{*} \otimes_{k} \mathcal{F}_{\mathcal{Y}_{0}(M)} \mathcal{E}_{\mathcal{C}_{0}\left(Y_{G} \cup W\right)}(N)\right) \\
& \subseteq\left\{V \in \mathcal{V}_{G}(k): V \subseteq Y_{G}\right\}
\end{aligned}
$$

where the final inclusion follows from Proposition 4.4. By Proposition 2.2, then, the module $\mathcal{F}_{\mathcal{Y}_{0}(M)} \mathcal{E}_{\mathcal{C}_{0}\left(Y_{G} \cup W\right)}(N)$ is in $\mathcal{C}_{0}\left(Y_{G}\right)^{\oplus}$, but it is also $\mathcal{C}_{0}\left(Y_{G}\right)$ local, and so must be projective.

As before, we easily deduce the following classification.

THEOREM 5.8. Let $G$ be a finite group, and let $\mathcal{C}$ be a thick subcategory of $\operatorname{stmod}_{0}(k G)$ that contains $\mathcal{C}_{0}\left(Y_{G}\right)$. Then $\mathcal{C}=\mathcal{C}_{0}(\mathcal{X})$ for some set $\mathcal{X}$ of closed homogeneous subvarieties of $V_{G}(k)$ that is closed under specialization and finite unions and includes $Y_{G}$.

6. Inside the nucleus. In this section we shall examine some examples of thick subcategories of $\operatorname{stmod}_{0}(k G)$ that are not covered by the theorems of Section 5. In particular, we shall consider thick subcategories of $\mathcal{C}_{0}\left(Y_{G}\right)$ for several groups with nontrivial nuclei.

In preparation for studying these examples, we begin with a theorem which applies in some of the cases we shall consider. For notation, let $\mathcal{N}$ denote the thick subcategory of $\operatorname{StMod}_{0}(k G)$ consisting of all modules $M$ with $\widehat{H}^{n}(G, M)=0$ for all $n$.

TheOREM 6.1. Suppose that $G$ has a normal subgroup $N$, and let $k$ be a field of characteristic $p$.

(i) Let $M$ be a $k G$-module with the property that $M \downarrow_{N}$ is projective and

$$
\operatorname{Hom}_{k N}\left(k, M \downarrow_{N}\right)=0 .
$$

Then $H^{*}(G, M)=0$, and hence $M$ is in $\mathcal{N}$.

(ii) Suppose that $G / N$ is a p-group. Let $M$ be an indecomposable and nonprojective module in the principal block, and suppose that for every nontrivial p-subgroup $Q \subseteq N, C_{N}(Q)$ is p-nilpotent. If $M$ is in $\mathcal{N}$ then $M \downarrow_{N}$ is projective and $\operatorname{Hom}_{k N}\left(k, M \downarrow_{N}\right)=0$. 
Proof. (i) The hypotheses imply that the $E_{2}$ page of the LyndonHochschild-Serre spectral sequence

$$
H^{*}\left(G / N, H^{*}(N, M)\right) \Rightarrow H^{*}(G, M)
$$

is zero, and so $H^{*}(G, M)=0$.

(ii) Since $G / N$ is a $p$-group, $M \downarrow_{N} \uparrow^{G} \cong k_{N} \uparrow^{G} \otimes M$ is in the thick subcategory generated by $M$, and so

$$
\widehat{H}^{*}\left(N, M \downarrow_{N}\right) \cong \widehat{H}^{*}\left(G, M \downarrow_{N} \uparrow^{G}\right)=0 .
$$

Hence, by Proposition 4.4, $M \downarrow_{N}$ is projective. Since $M$ is indecomposable and nonprojective, $\widehat{H}^{0}(G, M)=0$ implies $\operatorname{Hom}_{k G}(k, M)=0$, and hence $\operatorname{Hom}_{k N}\left(k, M \downarrow_{N}\right)^{G / N}=0$. Since $G / N$ is a $p$-group, this implies that $\operatorname{Hom}_{k N}\left(k, M \downarrow_{N}\right)=0$.

Example $1\left(C_{2} \times A_{4}\right.$ in characteristic two). Let $G=C_{2} \times A_{4}$, and let $k$ be an algebraically closed field of characteristic two. Then $k G$ has only one block, the principal block, and the only nontrivial subgroup of $G$ that does not have a 2-nilpotent centralizer is $C_{2} \times\{1\}$. The nucleus $Y_{G}$ therefore consists of a single line in $V_{G}(k)$, and so every nonprojective module in $\mathcal{C}\left(Y_{G}\right)$ has $Y_{G}$ as its variety. By Theorem 6.1 a $k G$-module is in $\mathcal{N}$ if and only if its restriction to $\{1\} \times A_{4}$ is projective and has no direct summand isomorphic to the projective cover of the trivial module.

Let $M$ be the $k G$-module which, as a module for $A_{4}$, is the projective cover $P_{k}$ of the trivial module, and on which $C_{2}$ acts trivially. In other words, $M$ is isomorphic to the permutation module $\operatorname{Ind}_{C_{2} \times C_{3}}^{G}(k)$. Then $M \cong \Omega M$.

Now consider the element

$$
\gamma=\sum_{x \in A_{4}} x
$$

of $k G$, which is a central element whose square is zero. For any $\lambda \in k$, there is an automorphism $\theta_{\lambda}$ of $k G$ that fixes $A_{4}$ and sends the generator $g$ of $C_{2}$ to $g+\lambda \gamma$.

We can now form an infinite family $\left\{M_{\lambda}: \lambda \in k\right\}$ of modules, where $M_{\lambda}$ is just the module $M$ with the $k G$-action twisted by the automorphism $\theta_{\lambda}$. That is, $M=U / V$ where $U=k G$. $u$ is the projective left ideal generated by $u=1+w+w^{2}$ for $w \in A_{4}$ an element of order three, and $V=k G((g-1) u-$ $\lambda \gamma)$. In particular, $M_{0}=M$. Since $M$ is periodic of period one, the same is true of all the $M_{\lambda}$. Also, as $M_{\lambda}$ is an indecomposable periodic module, $V_{G}\left(M_{\lambda}\right)$ must be a single line through the origin, which must in fact be the nucleus $Y_{G}$, since $M_{\lambda}$ is not projective on restriction to $C_{2} \times\{1\}$.

As modules for $A_{4}$, all of these modules are isomorphic to $P_{k}$, but instead of acting trivially, the generator $g$ of $C_{2}$ acts as id $+\lambda \phi$, where $\phi$ is a fixed nonzero $k A_{4}$-module homomorphism obtained as a composition

$$
P_{k} \rightarrow P_{k} / \operatorname{rad}\left(P_{k}\right) \rightarrow \operatorname{soc}\left(P_{k}\right) \rightarrow P_{k} .
$$


Proposition 6.2. For different values of $\lambda$, the modules $M_{\lambda}$ for $C_{2} \times A_{4}$ (in characteristic two) described above generate thick subcategories of $\mathcal{C}\left(Y_{G}\right)$ which intersect trivially.

Proof. Since the automorphisms of $k G$ involved in the definition of the modules satisfy

$$
\theta_{\lambda} \theta_{\mu}=\theta_{\lambda+\mu}
$$

for all $\lambda, \mu \in k$, it is sufficient to show that, for $\lambda \neq 0$, the thick subcategory generated by $M_{\lambda}$ intersects trivially with the thick subcategory generated by $M$.

By Frobenius reciprocity,

$$
\underline{\operatorname{Hom}}_{k G}\left(M, M_{\lambda}\right) \cong \underline{\operatorname{Hom}}_{k\left(C_{2} \times C_{3}\right)}\left(k, M_{\lambda} \downarrow_{C_{2} \times C_{3}}\right),
$$

which is zero (if $\lambda \neq 0$ ) since it is easy to check that there is only one indecomposable summand of $M_{\lambda} \downarrow_{C_{2} \times C_{3}}$ that has nonzero fixed points, and that this summand is projective.

Since $M \cong \Omega M$, it follows that $\underline{\operatorname{Hom}}_{k G}(X, Y)=0$ for every $X$ in the thick subcategory of $\operatorname{stmod}(k G)$ generated by $M$ and $Y$ in the thick subcategory generated by $M_{\lambda}$. So these subcategories must intersect trivially.

We can produce two more families of examples by simply taking the tensor product of the modules $M_{\lambda}$ with each of the two nontrivial simple $k G$-modules, which are both one-dimensional. The subcategories produced in this way have the property that they are contained in $\mathcal{N}$.

Remark. One might suspect that any thick subcategory with support in the nucleus is generated by

$$
\left\{M_{\lambda}: \lambda \in X\right\} \cup\left\{M_{\lambda} \otimes \omega: \lambda \in Y\right\} \cup\left\{M_{\lambda} \otimes \bar{\omega}: \lambda \in Z\right\}
$$

for suitable subsets $X, Y$ and $Z$ of $k$. Here, $\omega$ and $\bar{\omega}$ denote the two nontrivial simple $k G$-modules. If this were true, then any self-equivalence of the stable module category would permute the minimal elements of this collection of thick subcategories, namely those generated by a single $M$ or $M \otimes \omega$ or $M \otimes \bar{\omega}$. However, there is a self-equivalence of the derived category of $A_{4}$ that takes the projective cover $P_{k}$ of the trivial module to a complex

$$
\ldots \rightarrow 0 \rightarrow P_{\omega} \oplus P_{\bar{\omega}} \rightarrow P_{\omega} \oplus P_{\bar{\omega}} \rightarrow P_{k} \rightarrow 0 \rightarrow \ldots
$$

where $P_{\omega}$ and $P_{\bar{\omega}}$ are the other indecomposable projectives. This induces a self-equivalence of the stable module category of $C_{2} \times A_{4}$ that takes $M_{0}$ to a 20-dimensional indecomposable module whose restriction to $A_{4}$ is $P_{k} \oplus 2 P_{\omega} \oplus 2 P_{\bar{\omega}}$. The reader will find more details of some examples of thick subcategories given by self-equivalences of the derived category in Example 4. 
ExAmple $2\left(C_{2} \times A_{5}\right.$ in characteristic two $)$. Since the principal blocks of $A_{4}$ and $A_{5}$ are derived equivalent [17, Section 3] (i.e., the bounded derived categories of finitely generated modules for the two blocks are equivalent as triangulated categories), it follows from Theorem 2.1 of [16] that the principal blocks of $C_{2} \times A_{4}$ and $C_{2} \times A_{5}$ are also derived equivalent, and hence are stably equivalent by Corollary 2.2 of [15].

An infinite collection of thick subcategories that intersect trivially and have varieties contained in the nucleus can be obtained by taking the modules $N_{\lambda}$ that we get by applying this stable equivalence to the modules $M_{\lambda}$ given in the last example.

EXAMPLE 3 (Janko's sporadic group $J_{1}$ in characteristic two). Let $G$ be the sporadic group $J_{1}$ of Janko and let $k$ be an algebraically closed field of characteristic two. Then $G$ has just one conjugacy class of involutions, and the centralizer $H$ is isomorphic to $C_{2} \times A_{5}$. The nucleus $Y_{G}$ is equal to the image of $Y_{H} \rightarrow V_{G}(k)$, and is a single line through the origin in the three-dimensional variety $V_{G}(k)$.

By Theorem 4.2 of [3], induction gives an equivalence of categories between $\mathcal{C}\left(Y_{H}\right)$ and $\mathcal{C}\left(Y_{G}\right)$. It follows that the modules obtained by inducing $N_{\lambda}$ from $C_{2} \times A_{5}$ to $J_{1}$ generate thick subcategories of $\mathcal{C}\left(Y_{G}\right)$ which intersect trivially.

Proposition 6.3. For different values of $\lambda$, the modules $N_{\lambda}$ for $C_{2} \times A_{5}$ (in characteristic two) described above induce up to $J_{1}$ to give modules which generate thick subcategories of $\mathcal{C}\left(Y_{G}\right)$ intersecting trivially.

Example $4\left(C_{3} \times S_{3}\right.$ in characteristic three $)$. Now let

$$
G=C_{3} \times S_{3}=\left\langle x, y, z: x^{3}=y^{3}=z^{2}=1, x y=y x, x z=z x, y z=z y^{2}\right\rangle
$$

and let $k$ be an algebraically closed field of characteristic three. As in the previous example, $k G$ has only one block, and there is only one nontrivial subgroup (namely $C_{3} \times\{1\}$ ) whose centralizer is not 3-nilpotent, and so again the nucleus $Y_{G}$ is a single line through the origin. There are two simple $k G$-modules, $k$ and $\varepsilon$, both one-dimensional.

The following construction produces an infinite collection of thick subcategories of $\mathcal{C}\left(Y_{G}\right)$. Set $X=x-1$ and $Y=y^{2}-y$, so that $z X=X z$, $z Y=-Y z, X^{3}=0, X Y=Y X$, and $Y^{3}=0$. For $0 \neq \lambda \in k$, we define $M_{\lambda}$ to be the $k G$-module generated by elements $a$ and $b$, satisfying the following relations: $z a=-a, z b=-b, Y^{2} a=X^{2} b, \lambda X a=Y^{2} b$. Then $M_{\lambda}$ has a $k$-basis consisting of the elements $a, Y a, Y^{2} a=X^{2} b, b, Y b, Y^{2} b=\lambda X a$, $X b, X Y b$ and $X Y^{2} b=\lambda X^{2} a$.

The projective cover of $M_{\lambda}$ is a direct sum of two copies of $P_{\varepsilon}$ generated by elements $\widehat{a}$ and $\widehat{b}$ mapping to $a$ and $b$. The kernel is generated by $a^{\prime}=$ $Y^{2} \widehat{a}-X^{2} \widehat{b}$ and $b^{\prime}=\lambda X \widehat{a}-Y^{2} \widehat{b}$, which satisfy the same relations as $a$ and $b$. 
Counting dimensions, it follows that $\Omega M_{\lambda} \cong M_{\lambda}$, so that $M_{\lambda}$ is periodic with period one. In fact it can be seen that $M_{\lambda}$ is projective on restriction to $S_{3}$, and that $M_{\lambda}^{G}=0$ and $\operatorname{Hom}_{k G}\left(M_{\lambda}, k\right)=0$. So by Theorem 6.1 , every $M_{\lambda}$ is in $\mathcal{N}$.

If $M_{\mu}$ is another such module, with corresponding generators $c$ and $d$, we calculate $\operatorname{Hom}_{k G}\left(M_{\lambda}, M_{\mu}\right)$ as follows. The images of $a$ and $b$ must be in the -1 eigenspace of $z$, which has a $k$-basis $c, Y^{2} c=X^{2} d, d, Y^{2} d=\mu X c$, $X d$ and $X Y^{2} d=\mu X^{2} c$. Examining the relations in $M_{\lambda}$ and $M_{\mu}$, we obtain a system of linear equations which must be satisfied by these images. For $\lambda \neq \mu$, these imply that $\operatorname{Hom}_{k G}\left(M_{\lambda}, M_{\mu}\right)$ has a $k$-basis consisting of the following homomorphisms:

$$
\begin{array}{rlll}
\theta_{1}: & a \mapsto Y^{2} c=X^{2} d, & b \mapsto 0, \\
\theta_{2}: & a \mapsto \mu X^{2} c=X Y^{2} d, & b \mapsto 0, \\
\theta_{3}: & a \mapsto 0, & b \mapsto(\mu-\lambda) Y^{2} c, \\
\theta_{4}: & a \mapsto 0, & b \mapsto(\lambda-\mu) X c, \\
\theta_{5}: & a \mapsto 0, & b \mapsto \mu X^{2} c=X Y^{2} d, \\
\theta_{6}: & a \mapsto \mu X c=Y^{2} d, & b \mapsto \lambda X d .
\end{array}
$$

Each of these lifts to a map from $M_{\lambda}$ to the projective cover of $M_{\mu}$ as follows:

$$
\begin{array}{llll}
\widehat{\theta}_{1}: & a \mapsto X^{2} \widehat{d}, & b \mapsto 0, \\
\widehat{\theta}_{2}: & a \mapsto X Y^{2} \widehat{d}, & b \mapsto 0, \\
\widehat{\theta}_{3}: & a \mapsto 0, & b \mapsto \mu Y^{2} \widehat{c}-\lambda X^{2} \widehat{d}, \\
\widehat{\theta}_{4}: & a \mapsto \mu X^{2} \widehat{c}-X Y^{2} \widehat{d}, & b \mapsto \lambda X \widehat{c}-Y^{2} \widehat{d}, \\
\widehat{\theta}_{5}: & a \mapsto 0, & b \mapsto X Y^{2} \widehat{d}, \\
\widehat{\theta}_{6}: & a \mapsto Y^{2} \widehat{d}, & b \mapsto \lambda X \widehat{d} .
\end{array}
$$

It follows that $\underline{\operatorname{Hom}}_{k G}\left(M_{\lambda}, M_{\mu}\right)=0$ for $\lambda \neq \mu$. This, together with the fact that these modules are periodic of period one, implies that the modules $M_{\lambda}$ again generate thick subcategories which intersect trivially.

Proposition 6.4. For different values of $\lambda$, the modules $M_{\lambda}$ for $C_{3} \times$ $S_{3}$ (in characteristic three) described above generate thick subcategories of $\mathcal{C}\left(Y_{G}\right)$ which intersect trivially.

Here are some more examples of thick subcategories of $\operatorname{stmod}(k G)$ :

(a) The intersection of $\mathcal{C}\left(Y_{G}\right)$ with $\langle k\rangle$ or with $\langle\varepsilon\rangle$.

(b) Of course, $\mathcal{N}$ is a thick subcategory of $\mathcal{C}\left(Y_{G}\right)$. As noted before, all of the modules $M_{\lambda}$ are in $\mathcal{N}$. However, there are many more modules in $\mathcal{N}$ and may be many more thick subcategories of $\mathcal{N}$. 
(c) Let $L$ be the projective cover of the trivial module for $S_{3}$, regarded as a $k G$-module via inflation. Then $L$ has $Y_{G}$ as its variety, and so it generates a thick subcategory of $\mathcal{C}\left(Y_{G}\right)$. Since $\langle L, k\rangle$ contains $\varepsilon$, and must therefore be the whole of $\operatorname{stmod}(k G), L$ cannot be in $\langle k\rangle$. Also, $\underline{\operatorname{Hom}}_{k G}(k, L) \neq 0$, so $L$ is not in either of the categories (a) or (b).

(d) The modules $X$ of $\mathcal{C}\left(Y_{G}\right)$ for which $\underline{\operatorname{Hom}}_{k G}\left(\Omega^{n} L, X\right)=0$ for all $n \in \mathbb{Z}$ also form a nontrivial thick subcategory. One such module has the following Loewy structure:

$$
\begin{array}{ccccccccccccc}
\varepsilon & & & k & & & & k & & \varepsilon & & \\
\varepsilon & & k & & \varepsilon & & k & & \varepsilon & & k & \\
& k & & \varepsilon & & k & & \varepsilon & & k & & \varepsilon
\end{array}
$$

(e) One way of producing new examples of thick subcategories is to apply self-equivalences of $\operatorname{stmod}(k G)$ to old examples. By [15, Theorem 2.1], a selfequivalence of the derived category $D^{b}(\bmod (k G))$ induces a self-equivalence of the stable module category, and so the main theorem of [14] can be used to give examples.

For instance, if we denote by $Q_{k}$ and $Q_{\varepsilon}$ the projective covers of the two simple $k S_{3}$-modules, then let $T$ be the direct sum of the complex

$$
\ldots \rightarrow 0 \rightarrow Q_{\varepsilon} \rightarrow 0 \rightarrow 0 \rightarrow \ldots,
$$

concentrated in degree 1 , and the complex

$$
\ldots \rightarrow 0 \rightarrow Q_{\varepsilon} \rightarrow Q_{k} \rightarrow 0 \rightarrow \ldots,
$$

with a nonzero differential and concentrated in degrees 1 and 0 . It is easy to check that $T$ is a tilting complex [14, Definition 6.5] for $k S_{3}$ whose endomorphism ring is isomorphic to $k S_{3}$. Thus, by [14, Theorem 6.4], it induces a self-equivalence of $D^{b}\left(\bmod \left(k S_{3}\right)\right)$. The induced self-equivalence of $\operatorname{stmod}\left(k S_{3}\right)$ is actually isomorphic to the identity functor. However, by [16, Theorem 2.1], the tensor product $k C_{3} \otimes T$ is a tilting complex for $k G$ whose endomorphism ring is isomorphic to $k G$. In this case, the induced functor

$$
S: \operatorname{stmod}(k G) \rightarrow \operatorname{stmod}(k G)
$$

generates an infinite cyclic group of nontrivial self-equivalences of the cate$\operatorname{gory} \operatorname{stmod}(k G)$.

We can use this self-equivalence to produce more examples of thick subcategories of $\mathcal{C}\left(Y_{G}\right)$. For example, if $L$ is the module described in (c), then the modules $L, S(L)$ and $S^{2}(L)$ have Loewy structures of the form

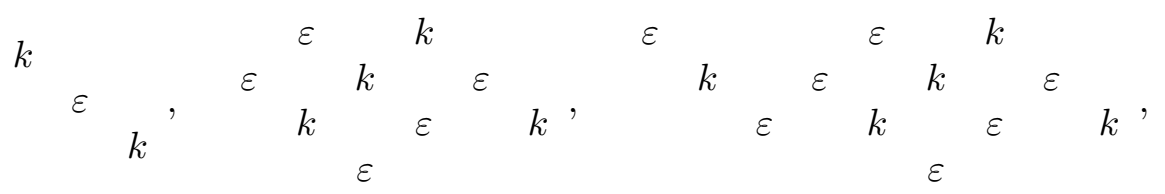


the structure of $S^{3}(L)$ is

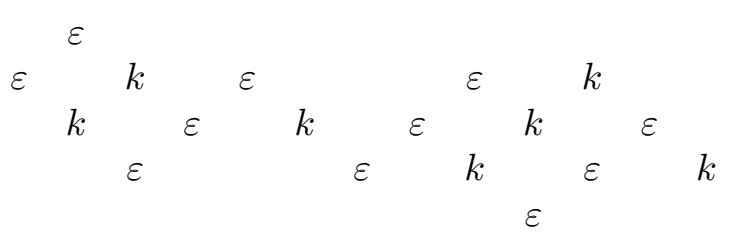

and so on. Also, $S^{-n}(L)$ is the dual of $S^{n}(L)$.

Each of these modules generates a thick subcategory of $\mathcal{C}\left(Y_{G}\right)$. Since $\underline{\operatorname{Hom}}_{k G}\left(\Omega^{n} \varepsilon, L\right)=0$ for all $n \in \mathbb{Z}$, but $\underline{\operatorname{Hom}}_{k G}\left(\varepsilon, S^{m} L\right)$ is nonzero for $m \neq 0$, all of these subcategories are different. Similarly, we can apply powers of $S$ to the thick subcategory described in (d).

(f) More examples can be constructed from those described above by taking the tensor product with $\varepsilon$.

EXAMPle $5\left(S L\left(2, \mathbb{F}_{3}\right)\right.$ in characteristic two). Let

$$
\begin{aligned}
G & =S L\left(2, \mathbb{F}_{3}\right) \cong Q_{8} \rtimes C_{3} \\
& =\left\langle x, y, z: z y=y^{-1} x=y x^{-1}, z^{3}=1, x z=z y, y z=z x y\right\rangle
\end{aligned}
$$

and let $k$ be an algebraically closed field of characteristic two. Then $k G$ has only one block, the principal block, and the only nontrivial subgroup of $G$ that does not have a 2-nilpotent centralizer is $Z(G) \cong C_{2}$. The nucleus $Y_{G}$ is the whole of $V_{G}(k)$, which is a single line through the origin. So every nonprojective module has $Y_{G}$ as its variety.

The following construction provides an infinite family of thick subcategories of $\mathcal{C}\left(Y_{G}\right)=\operatorname{stmod}(k G)$. Let $\omega, \bar{\omega}$ be the primitive cube roots of unity in $k$, so that $\omega+\bar{\omega}=1=\omega \bar{\omega}$, and set $X=x+\omega y+\bar{\omega} x y, Y=$ $x^{-1}+\bar{\omega} y^{-1}+\omega(x y)^{-1}$ in $k G$. Then $X^{2}=Y X Y, Y^{2}=X Y X, X^{2} Y=$ $X Y^{2}=Y^{2} X=Y X^{2}=0, z X=\omega X z$ and $z Y=\bar{\omega} Y z$. The group algebra $k Q_{8}$ of the subgroup $Q_{8}=\langle x, y\rangle$ has basis $1, X, Y, X Y, Y X, X Y X=Y^{2}$, $Y X Y=X^{2}, X Y X Y=Y X Y X=X^{3}=Y^{3}$. A basis for $k G$ can be obtained by multiplying these basis elements by $1, z$ and $z^{2}$. The advantage of this basis is that it is well adapted to the study of the simple modules, their extensions, the Loewy series of the group algebra, and so on.

For $0 \neq \lambda \in k$, let $M_{\lambda}$ be the $k G$-module with a single generator $a$, satisfying $z a=a$ and $X Y a=\lambda Y X a$. Thus $M_{\lambda}$ is four-dimensional over $k$, with basis $a, X a, Y a$ and $X Y a$. We have $X^{2} a=Y X Y a=\lambda Y^{2} X a=0$ and $Y^{2} a=X Y X a=\lambda^{-1} X^{2} Y a=0$. The action of $z$ is given by $z a=a$, $z X a=\omega X a, z Y a=\bar{\omega} Y a$ and $z X Y a=X Y a$. The projective cover of $M_{\lambda}$ is the eight-dimensional $k G$-module generated by a single element $\widehat{a}$ mapping to $a$ and satisfying $z \widehat{a}=\widehat{a}$. The kernel is generated by $a^{\prime}=(X Y+\lambda Y X) \widehat{a}$, 
and is also four-dimensional. Since

$$
\left(X Y+\lambda^{-1} Y X\right) a^{\prime}=\left(X Y X Y+\lambda X Y^{2} X+\lambda^{-1} Y X^{2} Y+Y X Y X\right) \widehat{a}=0,
$$

we have $\Omega\left(M_{\lambda}\right) \cong M_{\lambda-1}$.

If $M_{\mu}$ is another such module with corresponding generator $b$, and $\mu \notin$ $\left\{\lambda, \lambda^{-1}\right\}$, then $\operatorname{Hom}_{k G}\left(M_{\lambda}, M_{\mu}\right)$ is one-dimensional, generated by the homomorphism

$$
a \mapsto\left(\lambda+\mu^{-1}\right) X Y b=(\lambda X Y+Y X) b .
$$

This lifts to the homomorphism $a \mapsto(\lambda X Y+Y X) \widehat{b}$ to the projective cover of $M_{\mu}$, and so $\underline{\operatorname{Hom}}_{k G}\left(M_{\lambda}, M_{\mu}\right)=0$. It follows from this, together with the fact that $\Omega\left(M_{\lambda}\right) \cong M_{\lambda^{-1}}$, that the pairs $\left\{M_{\lambda}, M_{\lambda^{-1}}\right\}$ generate thick subcategories which intersect trivially, for different pairs $\left\{\lambda, \lambda^{-1}\right\}$.

Proposition 6.5. For different pairs $\left\{\lambda, \lambda^{-1}\right\}$, the pairs $\left\{M_{\lambda}, M_{\lambda^{-1}}\right\}$ for $S L\left(2, \mathbb{F}_{3}\right)$ (in characteristic two) generate thick subcategories of $\mathcal{C}\left(Y_{G}\right)$ which intersect trivially.

EXAmple $6\left(G L\left(2, \mathbb{F}_{3}\right)\right.$ in characteristic two). Let $G$ be the group

$$
G=G L\left(2, \mathbb{F}_{3}\right) \cong Q_{8} \rtimes S_{3},
$$

and let $H$ be the subgroup $S L\left(2, \mathbb{F}_{3}\right)$ discussed above. Let $k$ be an algebraically closed field of characteristic two. Then $k G$ again has only one block, and the only nontrivial subgroup of $G$ that does not have a 2-nilpotent centralizer is $Z(G)=Z(H) \cong C_{2}$. So the nucleus $Y_{G}$ is equal to $\operatorname{Im}\left(V_{H}(k) \rightarrow\right.$ $\left.V_{G}(k)\right)$, a single line through the origin in the affine plane $V_{G}(k)$.

We write $G$ as $H \rtimes\langle t\rangle$, with relations

$$
t^{2}=1, \quad x t=t x^{-1}, \quad y t=t(x y)^{-1}, \quad z t=t z^{-1} .
$$

Thus we have $X t=t Y$ and $Y t=t X$. It is easily seen that conjugation by $t$ swaps $M_{\lambda}$ with $M_{\lambda^{-1}}$. So $\widehat{M}_{\lambda}=M_{\lambda} \uparrow G \cong M_{\lambda^{-1}} \uparrow G$ is an indecomposable $k G$-module whose restriction to $H$ is isomorphic to $M_{\lambda} \oplus M_{\lambda^{-1}}$. It follows from Frobenius reciprocity that for $\mu \notin\left\{\lambda, \lambda^{-1}\right\}, \underline{\operatorname{Hom}}_{k G}\left(\widehat{M}_{\lambda}, \widehat{M}_{\mu}\right)=0$. Furthermore, $\Omega\left(\widehat{M}_{\lambda}\right) \cong \widehat{M}_{\lambda}$. So again, the modules $\widehat{M}_{\lambda}$ generate thick subcategories of $\operatorname{stmod}(k G)$ which intersect trivially for different pairs $\left\{\lambda, \lambda^{-1}\right\}$.

Proposition 6.6. For different pairs $\left\{\lambda, \lambda^{-1}\right\}$, the modules $\widehat{M}_{\lambda}$ for the group $G L\left(2, \mathbb{F}_{3}\right)$ (in characteristic two) generate thick subcategories of $\mathcal{C}\left(Y_{G}\right)$ which intersect trivially.

EXAMPLE $7\left(M_{11}\right.$ and $S L\left(3, \mathbb{F}_{3}\right)$ in characteristic two). Let $G$ be one of the groups $M_{11}$ or $S L\left(3, \mathbb{F}_{3}\right)$, and let $k$ be an algebraically closed field of characteristic two. It is well known that the principal blocks of these two 
groups are Morita equivalent $\left({ }^{1}\right)$, so we can discuss them simultaneously. The group $G$ has a single conjugacy class of involutions, and the centralizer $H$ is isomorphic to the group $G L\left(2, \mathbb{F}_{3}\right)$ discussed above. The nucleus $Y_{G}$ is equal to $\operatorname{Im}\left(Y_{H} \rightarrow V_{G}(k)\right)$, and is again a single line through the origin in the affine plane $V_{G}(k)$.

By Theorem 4.2 of [3], induction gives an equivalence of categories between $\mathcal{C}\left(Y_{H}\right)$ and $\mathcal{C}\left(Y_{G}\right)$. It follows that the modules $\widehat{M}_{\lambda} \uparrow^{G}$ generate thick subcategories of $\mathcal{C}\left(Y_{G}\right)$ which intersect trivially.

Proposition 6.7. For different pairs $\left\{\lambda, \lambda^{-1}\right\}$, the modules $\widehat{M}_{\lambda} \uparrow G$ for $M_{11}$ or $S L\left(3, \mathbb{F}_{3}\right)$ (in characteristic two) generate thick subcategories of $\mathcal{C}\left(Y_{G}\right)$ which intersect trivially.

7. Some open questions. We shall finish with some questions that remain unanswered. The most important, of course, is:

QUESTION 7.1. Is there a classification of the thick subcategories of the category stmod $k G$ for a general finite group G? Or, less ambitiously, what about the thick subcategories in the principal block?

In this paper, we have not looked at nonprincipal blocks, but perhaps the results of Section 5 could be generalized.

QUESTION 7.2. Is there an object associated to a nonprincipal block that plays the role that the nucleus $Y_{G}$ does for the principal block? If so, are there analogues of Theorems 5.5 and 5.8 ?

Returning to principal blocks, there are some more specific questions.

QUeSTION 7.3. For a group $G$ with a nontrivial nucleus $Y_{G}$, and a closed homogeneous subvariety $V$ of $Y_{G}$, is there always an infinite collection of thick subcategories of $\mathcal{C}(V)$ intersecting trivially, and each containing modules with variety $V$ ?

Given a thick subcategory $\mathcal{C}$ of $\operatorname{stmod}(k G)$, there is another thick subcategory whose objects are the modules $Y$ such that $\underline{\operatorname{Hom}}_{k G}(X, Y)=0$ for all $X$ in $\mathcal{C}$. Let us call this the perpendicular category $\mathcal{C}^{\perp}$ of $\mathcal{C}$. For example, $\langle k\rangle^{\perp}$ is the category $\mathcal{N}$ of modules $M$ for which $\widehat{H}^{*}(G, M)=0$. By "Tate duality" [1, Proposition 4.12.9] between $\underline{\operatorname{Hom}}(M, N)$ and $\underline{\operatorname{Hom}}(N, \Omega M)$, there is no difference between the "left perpendicular" and "right perpendicular" categories of a thick subcategory.

$\left({ }^{1}\right)$ The structure of the projective indecomposable modules for $M_{11}$ is given in Schneider's thesis [19]. Blocks with semidihedral defect groups are classified in Erdmann [9, 10], but there is a misprint on page 162 of [10], where it is stated that the principal block of $M_{11}$ is in family IV. In fact, it is in family III along with the groups $\operatorname{PSL}\left(3, \mathbb{F}_{q}\right)$ for $q \equiv 3$ modulo 4 . 
In the case when $G$ is a $p$-group and $\mathcal{X}$ is a collection of subvarieties closed under finite unions and specializations, $\mathcal{C}(\mathcal{X})^{\perp}$ is just $\mathcal{C}\left(\mathcal{X}^{\prime}\right)$, where

$$
\mathcal{X}^{\prime}=\left\{V \in \mathcal{V}_{G}(k): V \cap W=\{0\} \text { for all } W \in \mathcal{X}\right\} .
$$

It follows that $\mathcal{C}(\mathcal{X})^{\perp \perp}=\mathcal{C}(\mathcal{X})$ if and only if $\mathcal{X}$ is closed under arbitrary unions in the sense that if a subvariety $V$ is a union of elements of $\mathcal{X}$ then it is itself in $\mathcal{X}$.

QUeSTION 7.4. If $Y_{G}$ is not trivial, for which thick subcategories $\mathcal{C}$ of the category $\operatorname{stmod}(k G)$ does $\mathcal{C}=\mathcal{C}^{\perp \perp}$ ? In particular, is it always true that $\langle k\rangle=\langle k\rangle^{\perp \perp}$ ?

Even in the specific examples of Section 6, we do not know whether $\langle k\rangle=\langle k\rangle^{\perp \perp}$.

\section{References}

[1] D. J. Benson, Representations and Cohomology I, Cambridge Stud. Adv. Math. 30, Cambridge Univ. Press, 1990.

[2] - Representations and Cohomology II, Cambridge Stud. Adv. Math. 31, Cambridge Univ. Press, 1991.

[3] - Cohomology of modules in the principal block of a finite group, New York J. Math. 1 (1995), 196-205.

[4] D. J. Benson, J. F. Carlson and J. Rickard, Complexity and varieties for infinitely generated modules, Math. Proc. Cambridge Philos. Soc. 118 (1995), 223-243.

[5] - - - - Complexity and varieties for infinitely generated modules, II, ibid. to appear.

[6] D. J. Benson, J. F. Carlson and G. R. Robinson, On the vanishing of group cohomology, J. Algebra 131 (1990), 40-73.

[7] L. Chouinard, Projectivity and relative projectivity over group rings, J. Pure Appl. Algebra 7 (1976), 278-302.

[8] E. S. Devinatz, M. J. Hopkins and J. H. Smith, Nilpotence and stable homotopy theory, I, Ann. of Math. (2) 128 (1988), 207-241.

[9] K. Erdmann, Algebras and semidihedral defect groups I, Proc. London Math. Soc. 57 (1988), 109-150.

[10] —, Algebras and semidihedral defect groups II, ibid. 60 (1990), 123-165.

[11] M. J. Hopkins, Global methods in homotopy theory, in: Homotopy Theory (Durham, 1985), London Math. Soc. Lecture Note Ser. 117, Cambridge Univ. Press, 1987, 73-96.

[12] A. Neeman, Stable homotopy as a triangulated functor, Invent. Math. 109 (1992), $17-40$.

[13] - The chromatic tower for $D(R)$, Topology 31 (1992), 519-532.

[14] J. Rickard, Morita theory for derived categories, J. London Math. Soc. (2) 39 (1989), 436-456.

[15] - Derived categories and stable equivalence, J. Pure Appl. Algebra 61 (1989), 303-317.

[16] - Derived equivalences as derived functors, J. London Math. Soc. (2) 43 (1991), 37-48. 
[17] J. Rickard, Splendid equivalences: derived categories and permutation modules, Proc. London Math. Soc. (3) 72 (1996), 331-358.

[18] - Idempotent modules in the stable category, J. London Math. Soc., to appear.

[19] G. Schneider, Die 2-modularen Darstellungen der Mathieu-Gruppe $M_{12}$, doctoral dissertation, Essen, 1981.

Department of Mathematics

University of Georgia

Athens, Georgia 30602

U.S.A.

E-mail: djb@byrd.math.uga.edu jfc@sloth.math.uga.edu

Web: http://www.math.uga.edu/ djb/ http://www.math.uga.edu/ jfc/
School of Mathematics University of Bristol

University Walk

Bristol

BS8 1TW England

E-mail: J.Rickard@bristol.ac.uk

Received 11 October 1996;

in revised form 16 December 1996 